\title{
Hysteresis Loops for Magnetoelectric Multiferroics Using Landau-Khalatnikov Theory
}

\author{
Vincensius Gunawan, Ngurah Ayu Ketut Umiati \\ Physics Department, Faculty of Sciences and Mathematics, Diponegoro University, Indonesia
}

\begin{tabular}{l} 
Article Info \\
\hline Article history: \\
Received Mar 8, 2018 \\
Revised Jun 29, 2018 \\
Accepted Jul 22, 2018 \\
\hline
\end{tabular}

\section{Keyword:}

Cross interaction

Histeresis loops

Landau-Khalatnikov theory

Magnetoelectric multiferroics

Material response

\begin{abstract}
We present a theoretical discussion of the hysteresis in magnetoelectric multiferroics with bi-quadratic magnetoelectric coupling. The calculations were performed by employing Landau-Khalatnikov equation of motion for both the ferroelectric and ferromagnetic phase, then solve it simultaneously. In magnetoelectric, we obtain four types of hysteresis: ferroelectric hysteresis, ferromagnetic hysteresis and two types of cross hysteresis (electric field versus magnetization and magnetic field versus electric polarization). The cross hysteresis has butterfly shape which agree with the result from the previous research. It can also be seen from that hysteresis, that magnetization / electric polarization can not be flipped into the opposite direction using external electric / magnetic field when the magnetoelectric coupling is bi-quadratic type. Overall, the result shows that LandauKhalatnikov equation is able to approximate hysteresis loops in multiferroics system.
\end{abstract}

Copyright $@ 2018$ Institute of Advanced Engineering and Science. All rights reserved.

\section{Corresponding Author:}

Vincensius Gunawan,

Physics Department, Faculty of Sciences and Mathematics,

Diponegoro University,

Jl. Prof Soedarto, Tembalang, Semarang, Indonesia.

Email: goenangie@fisika.undip.ac.id

\section{INTRODUCTION}

Hysteresis is a phenomenon where the present condition is strongly depended on the past circumstance [1]. This trend can be found in functional material such as for example: magnetic material [2], [3], ferroelectrics [4] and semiconductors [5]. The hysteresis curves show the response of materials when an external stimulation is applied. In magnetics, a hysteresis curve illustrates the behavior of magnetization due to the applied external magnetic field. The hysteresis of ferroelectrics expresses the effect of an electric field to the polarization. Hence, the study of hysteresis of the functional materials is very important to understand the characteristic of the related materials. It is the basic step before functional materials can be utilized in many applications such as for example: measurement devices [6], field driven devices [7], LED devices [8], etc.

Magnetoelectric multiferroics are well known as material that possess both ferroelectricity and ferromagnetism at the same phase [9]. It means that the materials retain electric polarization and the magnetization which determine the properties of material. The ferroelectricity is related to the ferromagnetism through magnetoelectric coupling which responsible for the cross behaviour: the application of an electric field not only change the electric polarization in ferroelectrics phase, but also disturb the magnetization in magnetic phase [10]. In other way, the magnetic field can be used to drive the electric polarization [11]. As a consequence, there are four types of hysteresis for magnetoelectric multiferroics system. First, it is magnetic hysteresis relating magnetization (M) and magnetic field $\mathrm{H}$. Second type is the electric hysteresis connecting the electric polarisation $\mathrm{P}$ with the electric field $\mathrm{E}$. The third and fourth are magnetoelectric (cross-type) hysteresis linking $\mathrm{M}$ with $\mathrm{E}$ and $\mathrm{P}$ with $\mathrm{H}$. Considering the potential application 
of multiferroic materials (for example: electric field-controlled magnetic data storage [12]), the study of the hysteresis of this type of materials is unavoidable.

A numerical hysteresis loop for ferroelectrics is obtained by solving Landau-Khalatnikov (LKh) equation of motion which describes relaxation processes near phase transition [13], while numerical hysteresis for magnetic (ferromagnet) is acquired using Landau-Lifshitz-Gilbert (LLG) equation of motion which illustrates precession of the magnetization with the damping is considered [14], [15]. Hence, the numerical hysteresis for magnetoelectric multiferroics can be calculated using the electric LKh equation of motion and the magnetic LLG equation of motion simultaneously. However, the calculation by combining LKh and LLG equations of motion such in [16] is tough since LLG model requires more than one equation. Since obtaining hysteresis curve is basic step to understand the characteristic of the materials, it is better if the calculation to get hysteresis is less complicated.

Motivated by the facts that calculation using LKh theory is simpler than calculation employing LLG equation of motion and also there are some studies in magnetic system using approximation based on LKh equation of motion [17], [18], we propose in this numerical study to approach the problem of obtaining the hysteresis of the magnetoelectric multiferroics by employing Landau-Khalatnikov (LKh) theory for both systems (the ferroelectric and magnetic). In this paper, we want to show that this treatment is appropriate enough to give the initial feature, especially for obtaining the cross hysteresis in magnetoelectric system. As long as we know, there is no report regarding this treatment in multiferroics.

\section{RESEARCH METHOD}

The study begins by setting the energy density of the materials. Since magnetoelectric multiferroics comprise of ferroelectric and ferromagnet with magnetoelectric coupling, the energy density can be written

$$
F=F_{E}+F_{M}+F_{M E}
$$

where $F_{\mathrm{E}}, F_{\mathrm{M}}$ and $F_{\mathrm{ME}}$ represent the energy density for ferroelectric, ferromagnet phase and magnetoelectric interaction. Based on Landau phenomenological theory, the energy density of a system can be written as a polynomial of the order parameter of material. Here, the energy density for ferroelectric is represented by Ginzburg-Landau equation with electric polarization $(P)$ as order parameter expanded until quartic form as:

$$
F_{E}=\frac{1}{2} a P^{2}+\frac{1}{4} b P^{4}-E P
$$

with $a$ is temperature dependent constant defined as $a=a_{0}\left(T-T_{C}\right)$. Here, $a_{0}$ and $b$ in equation (2) represent dielectric stiffness constants while $E$ is an external electric field. Using similar procedure, we determine the energy density for ferromagnet with magnetization $(M)$ as the order parameter, in the form

$$
F_{M}=\frac{1}{2} \alpha M^{2}+\frac{1}{4} \beta M^{4}-H M
$$

where $\alpha=\alpha_{0}\left(T-T_{C}^{M}\right)$ is time dependent with $\alpha_{0}$ and $\beta$ are magnetic stiffness constants. Here $T_{C}^{M}$ is Curie temperature for magnet and $H$ represents an external magnetic field. According to symmetry argument, the energy density represents bi-quadratic coupling such as [19], [20]

$$
F_{M E}=-\frac{1}{2} \kappa P^{2} M^{2}
$$

where $\kappa$ represents magnetoelectric coupling constant, should always exist in all magnetoelectric multiferroics. The next step is performed by substituting the form of density energy equation (1) into the LandauKhalatnikov equation of motion

$$
\frac{\partial O_{i}}{\partial t}=-\gamma \frac{\partial F}{\partial O_{i}}
$$

where $\gamma$ is phenomenological damping constant and $O_{\mathrm{i}}$ represents the order parameter of the system. Since magnetoelectric multiferroics have electric polarization $(P)$ and magnetization $(M)$ as the order parameters, this step yields two coupled equations such as

$$
-\frac{1}{\gamma} \frac{\partial P}{\partial t}=a P+b P^{3}-\kappa P M^{2}-E
$$


for ferroelectric phase, and

$$
-\frac{1}{\gamma_{m}} \frac{\partial M}{\partial t}=\alpha M+\beta M^{3}-\kappa P^{2} M-H
$$

for ferromagnet phase.

Using rescalling processes, we can write LKh equation of motions in equation (6) with the dimensionless polarization $p$ and dimensionless magnetization $m$. Then, the rescalled equations can be written in the form which is appropriate for numerical calculations as

$$
(1-\gamma a \Delta t) p_{i+1}+\gamma a \Delta t p_{i+1}^{3}+\gamma a \Delta t K m_{i+1}^{2} p_{i+1}-p_{i}-\gamma a \Delta t \varepsilon_{i+1}=0
$$

and

$$
\left(1-\gamma_{m} \alpha \Delta t\right) m_{i+1}+\gamma_{m} \alpha \Delta t m_{i+1}^{3}+\gamma_{m} \alpha \Delta t K p_{i+1}^{2} m_{i+1}-m_{i}-\gamma a \Delta t h_{i+1}=0
$$

where $P=P_{\mathrm{s}} p, M=M_{\mathrm{s}} m$ with $P_{\mathrm{s}}$ and $M_{\mathrm{s}}$ represent the spontaneous electric polarization and lattice magnetization. The external electric field, the magnetic field and ME coupling are modified as, $\mathcal{E}=E\left(\frac{b}{a^{3}}\right)^{\frac{1}{2}}, m=H\left(\frac{\beta}{\alpha^{3}}\right)^{\frac{1}{2}}$ and $K=\left(\frac{a \alpha}{b \beta}\right) \kappa$. Here, the subscript of order parameter is related to the time as $E_{i}=E(t)$ and $E_{i+1}=E(t+\Delta t)$. The last step is solving the coupled LKh equation in equation (7a) and (7b) numerically. In this process, the electric $(p-\mathcal{E})$ hysteresis and cross $(m-\mathcal{E})$ hysteresis can be obtained by setting the external magnetic field to zero while variating the electric field. On the other hand, by setting the external electric field to zero while variating the magnetic field, we can get magnetic $(m-h)$ hysteresis and cross $(p-h)$ hysteresis.

In this research, we also study the role of magnetoelectric coupling in affecting the shape of cross hysteresis curve. It can be performed by solving the hysteresis formulas in several values of the magnetoelectric coupling. In practice, changing the values of the coupling is very difficult. However, the result can be used to predict the strength of the magnetoelectric coupling when the measurement of the cross hysteresis is performed.

\section{RESULTS AND ANALYSIS}

In this numerical calculation, we set $\frac{a}{b}=10^{10} \mathrm{statC}^{2} / \mathrm{cm}^{4} ; \frac{\alpha}{\beta}=10^{9} \mathrm{Oe}^{2}$, typical for ferroelectric and ferromagnetic phase. We also keep the value $\Delta t=0.05 ; \gamma_{m} \alpha \Delta t=0.005$ and scalled magnetoelectric constant $K=5 \times 10^{3}$. Solutions of the LKh equations of motion in equation (7) are illustrated in Figure 1 . We obtained four types of hysteresis: $(p-e),(m-h),(p-h)$ and $(m-e)$ hysteresis. The hysteresis for $(p-e)$ in Figure 1(a) and $(m-h)$ in Figure 1(c) have well known symmetrical shape where the electric polarisation $p$ and the magnetic magnetization $m$ have range from negative to positive values. Hence, both types of hysteresis posses coercive fields which are required to bring the electric polarization or magnetization to zero value. It means that the electric polarization $p$ is able to be flipped into the opposite direction using an external electric field $e$, while the magnetization $m$ can be flipped by applying an external magnetic field $h$. These $(p-e)$ and $(m-h)$ hysteresis curves have similar shape to the hysteresis for ferroelectric materials and ferromagnet materials from the earlier researches.

The calculated cross $(m-\varepsilon)$ and $(p-h)$ hysteresis which is the most important finding are shown in Figure 1(b) and (d). We found that the shape of hysteresis curves is very diferent compare to the shape of $(p-e)$ and $(m-h)$ hysteresis. These cross $(m-\varepsilon)$ and $(p-h)$ hysteresis posses butterfly shape with the range of the electric polarization or magnetization is only involving positive values. These numerical results qualitatively agree to the results from the previous experimental reports [21], [22]. The butterfly hysteresis curves in Figure 1(b) and (d) do not have coercive field since the electric polarization or magnetization could not be brought to zero value. It means that the electric polarization and magnetic magnetization in these butterfly shape curves in Figure 1(b) and (d) could not be flipped into the opposite direction. We argue that it occurs since bi-quadratic type of magnetoelectric coupling does not sensitive to the direction of the applied field. Both of the magnetization and the electric polarization are in quadratic term, means that the strength of that parameters which is important and not the direction of the electric polarization or magnetization. 


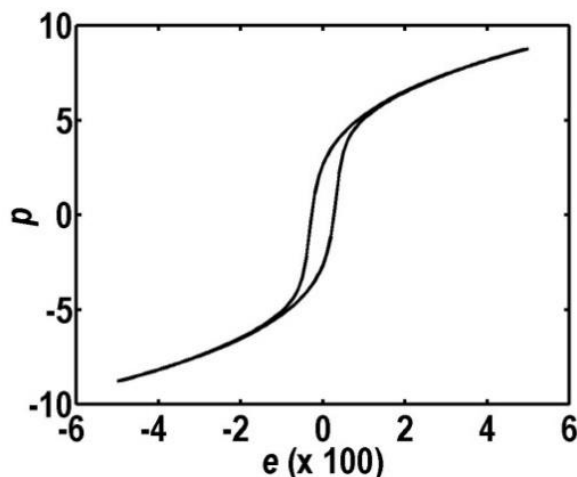

(a)

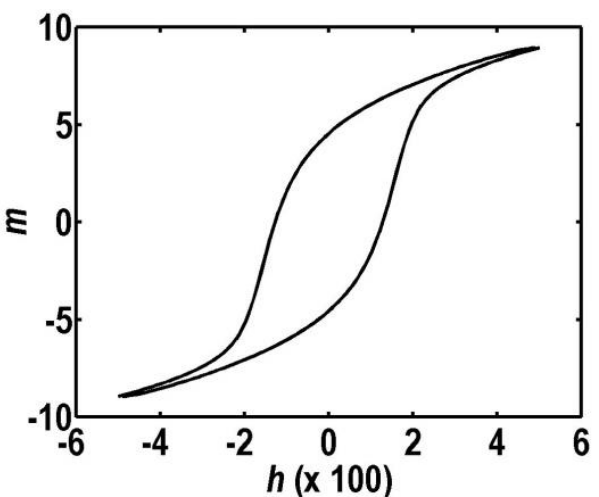

(c)

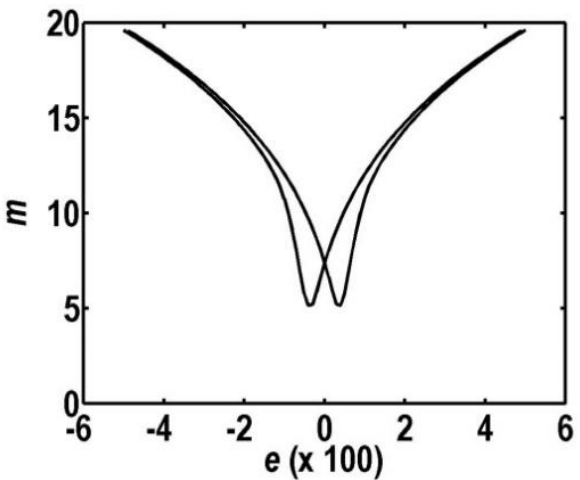

(b)

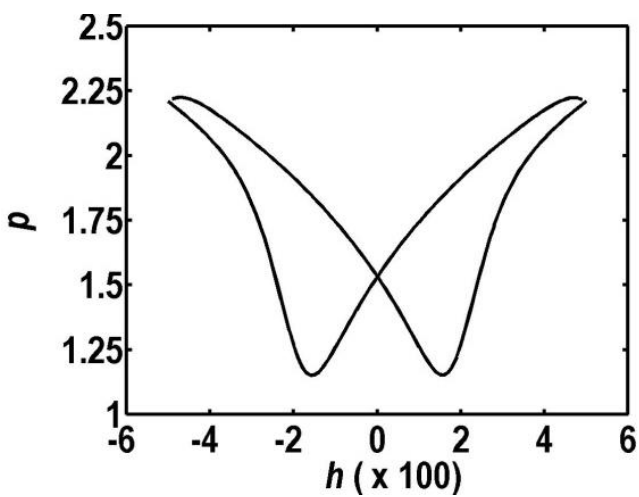

(d)

Figure 1. The hysteresis of magnetoelectric multiferroics with bi-quadratic couplings. Figure (a) and (c) are illustrating the electric $(p-e)$ hysteresis and the magnetic $(m-h)$ hysteresis. The other curves showing "cross" hysteresis where (b) displays the response of magnetization to the applied electric field while (d) indicates the response of polarization to the applied magnetic field

We also study the role of the strength of magnetoelectric coupling in determining the crosshysteresis in multiferroics materials. The results are shown in Figure 2 with the strength of the coupling in Figure 2(a) and Figure 2(c) is weaker than the coupling in Figure 2(b) and Figure 2(d) by factor $10^{-2}$. It can be seen that the response of the magnetization or the electric polarization to the applied electric or magnetic fields are weak when the strength of the magnetoelectric is small. It is clear when the electric/magnetic field is applied to the magnetoelectric (ME) multiferroics, it will change the state of the electric/magnetic system significantly. If the ME coupling is small, then these changes of the electric/magnetic system will only give the small disturbance to the magnetic/electric phase which in turn resulting weak responses of the magnetization/electric polarization. This agrees with the general feature of magnetoelectric materials: the cross effect is hardly to sense if the magnetoelectric coupling is weak.

Overall, the results stated that the approach using Landau-Khalatnikov equation is able to produce a qualitatively good prediction. The shape of cross hysteresis evidently similar to the previous experiment study. The approximation also yields reasonably feature regarding to the strength of the coupling. 


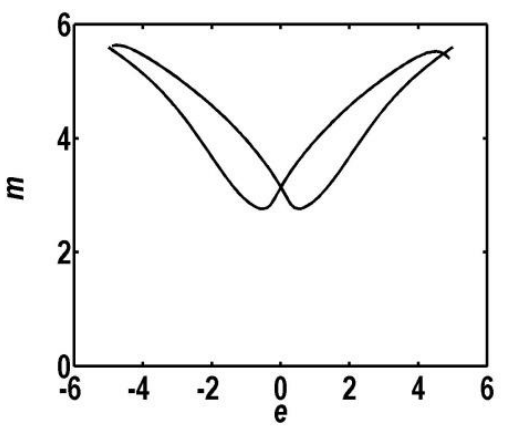

(a)

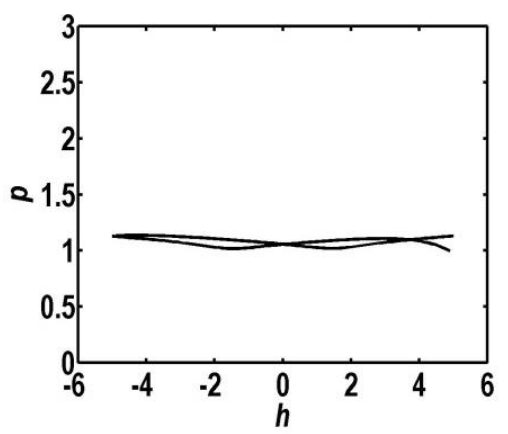

(c)

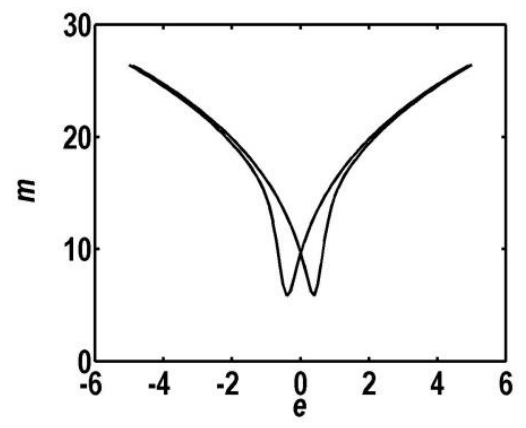

(b)

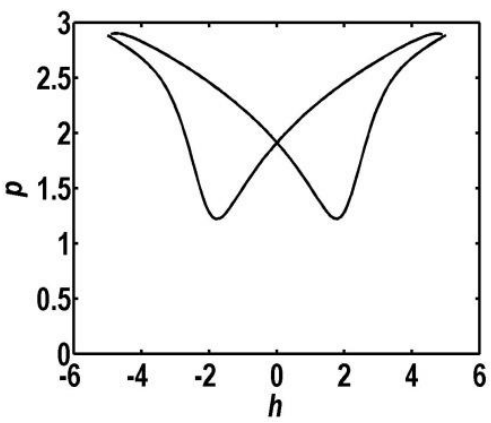

(d)

Figure 2. The cross hysteresis of magnetoelectric multiferroics with bi-quadratic couplings. Figure (a) and (b) are showing the cross $(m-e)$ hysteresis. The bi-quadratic coupling in (a) is smaller by factor $10^{-2}$ than the coupling in (b). The similar patern is also illustrated in (c) and (d) for cross $(p-h)$ hysteresis. Here, the strength of magnetoelectric coupling in (c) is $10^{-2}$ times weaker than that in (d)

\section{CONCLUSION}

In this research, we prove that Landau-Khalatnikov equation of motion may be used entirely for both ferroelectric and ferromagnetic phase to approximate the hysteresis of the magnetoelectric multiferroics with bi-quadratic phase. The approximation is able to produce the 'butterfly-shape' for cross $(p-h)$ and $(m-e)$ hysteresis which agree to the previous experiment result. Hence, this approximation is adequate to initial study to get the outline feature of the system before we perform the detail treatment using Landau-LifshitzGilbert equation for spin dynamics in magnetic system.

\section{ACKNOWLEDGEMENTS}

This study is a part of the research project in the topic of "the TM polaritons generated in multiferroics". We acknowledge Faculty of Sciences and Mathematics, Diponegoro University for funding this reseach through the contract No. 1646N/UN7.5.8/PP/2017.

\section{REFERENCES}

[1] D. H. Everett and W. I. Whitton, “A General Approach to Hysteresis,” Trans. Faraday Soc., vol. 48, pp. 749-757, 1952.

[2] D. C. Jiles, et al., "Numerical Determination of Hysteresis Parameters for the Modelling of Magnetic Properties using The Theory of Ferromagnetic Hysteresis," IEEE Trans. Mag., vol/issue: 28(1), pp. 27-35, 1992.

[3] D. Lin, et al., "Improved Vector Play Model and Parameter Identification for Magnetic Hysteresis Materials," IEEE Trans. Mag., vol/issue: 50(2), pp. 7008704, 2014.

[4] S. Sivasubramanian, et al., "Physical Kinetics of Ferroelectric Hysteresis," Ferroelectrics., vol/issue: 300(1), pp. 43$55,2010$.

[5] J. S. Lee, et al., "Two Opposite Hysteresis Curves in Semiconductors with Mobile Dopants," App. Phys. Lett., vol. 102, pp.253503, 2013.

[6] H. M. Luqman, et al., "Planning and Conducting Magnetic Field Level Measurement from Overhead Transmission Line," International Journal of Electrical and Computer Engineering (IJECE), vol/issue: 7(6), pp. 3124-3132, 2017.

[7] M. Boujemaa and C. Rachid, "Field Oriented Control of PMSM Supplied by Photovoltaic Source," International Journal of Electrical and Computer Engineering (IJECE), vol/issue: 6(3), pp. 1233-1247, 2016. 
[8] M. W. Umar, et al., "PWM Dimming Control for High Brightness LED Based Automotive Lighting Applications," International Journal of Electrical and Computer Engineering (IJECE), vol/issue: 7(5), pp. 2434-2440, 2017.

[9] H. Schmid, "Multi-ferroic Magnetoelectrics," Ferroelectrics, vol/issue: 162(1), pp. 317, 1994.

[10] Y. Chu, et al., "Electric Field Control of Local Ferromagnetism using a Magnetoelectric Multiferroics," Nat. Mat, vol. 7, pp. 478-482, 2008.

[11] T. Kimura, et al., "Magnetic Control of Ferroelectric Polarization." Nature, vol. 46, pp. 55-58, 2003.

[12] N. A. Spaldin and M. Fiebig, "The Renaissance of Magnetoelectric Multiferroics," Science, vol.309, pp.391-392, 2005.

[13] L. Cui, et al., "Landau-Khalatnikov Theory for The Hysteresis Loops of a Ferroelectric Thin Film," Chinese Journal of Physics, vol/issue: 52(3), pp. 1091-1099, 2014.

[14] M. Laksmanan, "The Fascinating World of The Landau-Lifshitz-Gilbert Equation: An Overview," Phil. Trans. R. Soc. A, vol. 369, pp. 1280-1300, 2011.

[15] E. A. Jagla, "Hysteresis Loops of Magnetic Thin Films with Perpendicular Anisotropy," Phys. Rev. B, vol/issue:72(9), pp. 294406, 2005.

[16] A. Sukhov, et al., "Polarization and Magnetization Dynamics of a Field-driven Multiferroic Structure," J. Phys.: Condens Matter, vol.22, pp. 352201, 2010

[17] A. P. Kamantsev, et al., "Thermodynamic and Relaxation Processes near Curie Point in Gadolinium," Solid State Phenomena, vol.215, pp.113-118, 2014.

[18] C. Binek, "Training of The Exchange Bias Effect: A Simple Analytic Approach,” Phys. Rev. B, vol.70, pp. 014421, 2004.

[19] A. Planes, et al., "Thermodynamics of Multicaloric Effects in Multiferroics," Phil. Mag, vol/issue: 94(17), pp. 1893$1908,2014$.

[20] M. Daraktchiev, et al., "Landau Theory of Domain Wall Magnetoelectricity," Phys. Rev. B., vol/issue: 81(22), pp. $224118,2010$.

[21] C. Israel, et al., "Converse Magnetoelectric Coupling in Multilayer," Appl. Phys. Lett., vol/issue: 93(17), pp.173501, 2008.

[22] J. F. Scott, “Application of Magnetoelectrics,” J. Matter. Chem., vol. 22, pp. 4567-4574, 2012.

\section{BIOGRAPHIES OF AUTHORS}

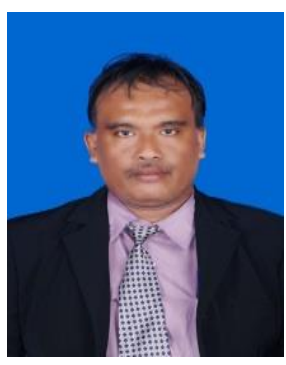

Vincensius Gunawan received the B.Sc and M.Sc degrees in Physics from Gadjah Mada University in 1995 and 2003, respectively. He then obtained his PhD in Physics from The University of Western Australia in 2012. He is currently working in Physics Department, Diponegoro University. His research interest includes the areas of polaritons, ferroelectrics, magnetics and multiferroics.

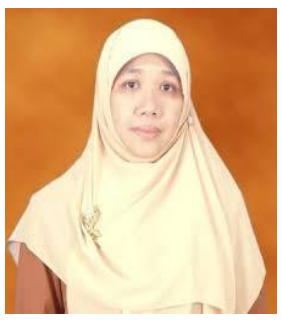

Ngurah Ayu Ketut Umiati received B.Sc degree in Physics from Diponegoro University in 1998. Later, she get her M.Sc degree in Physics from Bandung Institute of Technology in 2001. She recently obtained her PhD in Physics from Gadjah Mada University in 2016. She is now working in condensed matter group, Physics Department, Diponegoro University. Her research interest includes the area of ferroelectrics, dielectrics and plasmon-polimer. 